\title{
Correction to: An Integrated Modelling Approach for Flood Simulation in the Urbanized Qinhuai River Basin, China
}

\author{
Runjie $\mathrm{Li}^{1,2}$ • Jinkang $\mathrm{Du}^{1,2} \cdot$ Guodong Bian ${ }^{3,4} \cdot$ Yuefeng Wang ${ }^{5} \cdot$ Changchun Chen $^{6}$. \\ Xueliang Zhang ${ }^{1,2} \cdot$ Maohua $\mathrm{Li}^{1,2} \cdot$ Shanshan Wang ${ }^{1,2} \cdot$ Senyao $\mathrm{Wu}^{1,2}$. \\ Shunping $\mathrm{Xie}^{1,2} \cdot$ Long Yang $^{1} \cdot$ Chong-Yu Xu $^{7}$
}

Published online: 8 September 2020

(C) Springer Nature B.V. 2020

\section{Correction to: Water Resour Manag. https://doi.org/10.1007/s11269-020-02614-4}

The original version of this article contains incorrect symbol in eq. 7 and incorrect font style in eq. 8 which have been corrected.

The corrected version of eq. 7 should be $R_{i}=\varphi \times P$.

The correccted version of eq. 8 should be $R_{d}=P-\left(W M-W_{0}\right)-E$.

Publisher's Note Springer Nature remains neutral with regard to jurisdictional claims in published maps and institutional affiliations.

The online version of the original article can be found at https://doi.org/10.1007/s11269-020-02614-4

Long Yang

yanglong@nju.edu.cn

1 School of Geography and Ocean Science, Nanjing University, Nanjing, Jiangsu Province 210023, China

2 Jiangsu Center for Collaborative Innovation in Geographical Information Resource Development and Application, Nanjing, Jiangsu Province 210023, China

3 State Key Laboratory of Water Resources and Hydropower Engineering Science, Wuhan University, Wuhan 430072, China

4 Nanjing Hydraulic Research Institute, Nanjing 210029, China

5 School of Geography and Tourism, Chongqing Normal University, Chongqing 401331, China

6 School of Geographical Sciences, Nanjing University of Information Science and Technology, Nanjing, Jiangsu Province 210044, China

7 Department of Geosciences, University of Oslo, PO Box 1047, N-0316 Oslo, Blindern, Norway 